Pacific Journal of Mathematics

BI-INVARIANT PSEUDO-LOCAL OPERATORS ON LIE 


\section{BI-INVARIANT PSEUDO-LOCAL OPERATORS ON LIE GROUPS}

\section{Preiss Rothschild}

Let $G$ be a connected Lie group whose Lie algebra is not the semi-direct sum of a compact algebra and a solvable algebra. It is shown that any bi-invariant pseudo-local operator on $G$ is the sum of an invariant differential operator and an operator with smooth kernel.

1. Introduction. We consider a class of operators on Lie groups, satisfying a weak local property. Roughly, a pseudo-local operator on a manifold $M$ is a continuous linear operator, $P$, on the space of compactly supported functions on $M$, which extends to an operator $P^{\prime}$ on the space of compactly supported distributions on $M$, such that $P^{\prime}$ preserves singular support. It has been shown by Kohn and Nirenberg [3] that any pseudo-differential operator is pseudo-local. Stekaer [6] has proved that any bi-invariant pseudo-local operator on a complex semisimple Lie group is the sum of an invariant differential operator and an operator with smooth kernel. The proof of this theorem reduces to verifying that every smooth, invariant function on the Lie algebra of $G$ minus the origin can be extended smoothly over the origin. Our main result is the verification of this hypothesis for a large class of Lie groups, proving the above theorem for these groups. For a given Lie group, this theorem implies that the class of bi-invariant differential operators on that group can be substantially extended only by considering operators which do not satisfy local properties.

After the original version of this paper had been submitted, the author learned that these results have been extended by Anders Melin [8] to include any Lie algebra which is not the direct sum of a compact algebra and an abelian one. ${ }^{1}$ Independently, the author had extended the results to include the nilpotent case.

The author wishes to thank I. M. Singer, Victor Guillemin, and Gerald McCullom for helpful discussions on this work, and the referee for many suggestions which have greatly improved the exposition.

2. Definitions and notation. Let $G$ be a Lie group and $C^{\infty}(G)$, (resp. $C_{0}^{\infty}(G)$ ), the space of smooth functions (resp. smooth functions with compact support) on $G$. The dual of $C^{\infty}(G)$, which is the space of compactly supported distributions on $G$, will be denoted $\mathscr{E}^{\prime}(G)$, while the dual of $C^{\infty}(G)$, the space of distributions on $G$, will be denoted $\mathscr{D}^{\prime}(X)$.

1. The author is indebted to Sigurdur Helgason for informing her of Melin's work. 
For $u \in \mathscr{E}^{\prime}(X)$ we define the singular support of $u$, denoted sing supp $u$, as $\left\{x \in X \mid u \notin C^{\infty}(U)\right.$ for any neighborhood $U$ of $\left.x\right\}$.

A continuous linear operator $P: C_{0}^{\infty}(X) \rightarrow C^{\infty}(X)$ is called a pseudolocal operator if it extends to a continuous operator $P^{\prime}: \mathscr{E}^{\prime}(X) \rightarrow \mathscr{D}^{\prime}(X)$ such that $P^{\prime}$ preserves singular support; that is,

$$
\text { sing supp } P^{\prime} u \subseteq \operatorname{sing} \operatorname{supp} u \quad \text { for } u \in \mathscr{E}^{\prime}(X) \text {. }
$$

We now assume that there is a Lie group $G$ which operates differentiably on $X$. That is, there is a differentiable map $z$

$$
z: G \times X \rightarrow X
$$

such that $z(a b, x)=z(a, z(b, x))$ for all $a, b \in G$, and all $x \in X$. If $f \in$ $C_{0}^{\infty}(X)$ we define ${ }_{a} f$, the left translate of $f$ by $a \in G$ as

$$
{ }_{a} f(X)=f(z(a, x)) \quad \text { for } x \in X \text {. }
$$

If $X=G$, then the right translate of $f$ by $a \in G$ is defined by

$$
f_{a}(b)=f(b a) \quad \text { for } a \in G \text {. }
$$

We call the pseudo-local operator $P$ left invariant (resp. right invariant) if

$$
\left.P\left({ }_{a} f\right)={ }_{a}(P f) \quad f \in C_{0}^{\infty}(X) \quad \text { (resp. } P\left(f_{a}\right)=(P f)_{a}\right) .
$$

If $G=X$ is a Lie group, $P$ is called bi-invariant if it is both left and right invariant.

Let $\mathfrak{g}$ be the Lie algebra of $G$, and let 1 denote the identity in $G$. $G$ acts a group of automorphisms on $g$ via the adjoint representation, $A d$. For any $a \in G, x \in g$, we write $a . x$ for $A d(a) x$. A function $f$ on $g$ or $g-\{0\}$ is called invariant if it is constant on the orbits of $G$ on $g$. A function on $G$ or $G-\{1\}$ is called invariant if it is constant on the conjugacy classes of $G$. If $\mathscr{F}$ is a family of functions, $\mathscr{F}^{a}$ will denote the subset of invariant functions.

3. Pseudo-local operators on Lie groups. Our main result is the following.

THEOREM. Let $G$ be a connected Lie group and $\mathrm{g}$ its Lie algebra. If $\mathrm{g}$ is not the semi-direct sum of a compact algebra and a solvable one, then every bi-invariant pseudo-local operator on $G$ is the sum of an invariant differential operator and an operator with smooth kernel.

This theorem has been proved by Stekaer-Hansen in the special case where $\mathfrak{g}$ is complex reductive, non-abelian, using the following reduction to a criterion involvling invariant functions on $\mathrm{g}$. 
Proposition 1 (Stetkaer). Let $G$ be a connected Lie group with Lie algebra g. If the restriction map $r:\left(C^{\infty}(\mathfrak{g})\right)^{G} \rightarrow\left(C^{\infty}(\mathfrak{g}-\{0\})^{G}\right.$ is surjective, then every bi-invariant pseudo-local operator on $G$ is the sum of an invariant differential operator and an operator with smooth kernel.

For the proof of this proposition see Stetkaer [6].

We shall refer to the condition on $\mathfrak{g}$ in the proposition as Stetkaer's hypothesis.

Stetkaer's verification of this hypothesis for the case where $\mathfrak{g}$ is complex reductive uses a result of Kostant ([4] Theorem 7). Kostant's theorem implies the existence of a hyperplane $\mathfrak{v} \subset \mathfrak{g}-\{0\}$ and a smooth map $t: \mathfrak{g} \rightarrow \mathfrak{b}$ satisfying the following conditions.

(i) There is a dense subset $\mathfrak{x} \subset \mathfrak{g}$ such that for any $x \in \mathfrak{r}$, there exists a unique $x^{\prime} \in \mathfrak{b}$ with $a . x=x^{\prime}$ for some $a \in G$.

(ii) For any $x \in \mathfrak{x}, t(x)=x^{\prime}$. In particular, if $y \in \mathfrak{v} \cap \mathfrak{x}$, then $t(y)=y$. Conditions (i) and (ii) above show that any invariant function $f$ on $\mathfrak{g}-\{0\}$ is completely determined by its values on $\mathfrak{v}$. Since $\mathfrak{v} \subset \mathfrak{g}-$ $\{0\}$, the function $f \circ t$ is defined and smooth on all of $g$. Therefore $f \circ t$ is the desired extension of $f$ since in agrees with $f$ on $g-\{0\}$.

Since Kostant's result does not extend even to real reductive Lie groups, we shall use a substantially different approach in our proof.

4. Proof of the main theorem. We shall verify Stetkaer's hypothesis in the case where $g$ is not the semi-direct sum of a compact Lie algebra and a solvable Lie algebra. If $f$ is an invariant function which is smooth on $\mathfrak{g}-\{0\}$, it will be shown by explicit computation that all partial derivatives of $f$ can be extended continuously over 0 . We shall define a one parameter subgroup $\left\{a_{t}\right\}_{t \in \boldsymbol{R}}$ of $G$ and show that for a suitable basis of $\mathfrak{g}$ the transformation of the partial derivatives with respect to this basis can be easily computed (Lemma 3). Invariance of $f$ under the action of this one-parameter group is sufficient to prove the theorem, since the action of $\left\{a_{t}\right\}_{t \in R}$ pushes "most" small elements in $g-\{0\}$ to the unit sphere.

Let $\mathfrak{g}=\mathfrak{g}_{1}+\mathfrak{g}_{2}$ be a Levi decomposition of $\mathfrak{g}$, with $\mathfrak{g}_{1}$ semisimple, and $g_{2}$ solvable. By assumption, $\mathfrak{g}_{1}$ is not compact. From the structure theory of semisimple algebras, it is well known that $\mathfrak{g}_{1}$ contains a subalgebra $\mathfrak{t}$, where $\mathfrak{H}$ is isomorphic to $\mathfrak{I l}(2, R)$, the Lie algebra of the real special linear group. (For the proof of this, as well as the details of the representation theory of $\mathfrak{t}$, to be used later, see 
Serre (5), Chapitre IV and VI) or Helgason ([1] Chapter VI].) For any $x \in \mathfrak{g}$ let $a d x$ be the endomorphism defined by ad $x(y)=[x, y]$ for all $y \in g$; i.e. $a d$ is the adjoint representation. If $\mathfrak{t}$ is any such subalgebra, let $x \in \mathfrak{t}$ be the inverse image of the element $\left(\begin{array}{rr}1 & 0 \\ 0 & -1\end{array}\right)$ under a fixed isomorphism of $\mathfrak{a}$ with $\mathfrak{I}(2, \boldsymbol{R})$. From the representation theory of three-dimensional simple Lie algebras there is a vector sum decomposition

$$
\mathfrak{g}=\mathfrak{g}^{(0)}+\sum_{j=1}^{k} \mathfrak{g}^{\left(r_{j}\right)}+\sum_{j=1}^{k} \mathfrak{g}^{\left(-r_{j}\right)},
$$

where $\mathfrak{g}^{\left(r_{j}\right)}=\left\{y \in \mathfrak{g} \mid[x, y]=r_{j} y\right\}, \mathfrak{g}^{\left(-r_{j}\right)}=\left\{y \in \mathfrak{g} \mid[x, y]=-r_{j} y\right\}, \mathfrak{g}^{(0)}=$ $\{y \in \mathfrak{g} \mid[x, y]=0\}$ where the $r_{j}$ are all positive integers.

$$
\text { Let } \mathfrak{g}^{+}=\sum_{j=1}^{k} \mathfrak{g}^{\left(r_{j}\right)} \text { and } \mathfrak{g}^{-}=\sum_{j=1}^{k} \mathfrak{g}^{\left(-r_{j}\right)} \text {. }
$$

We make the convention that $r_{-j}=-r_{j}$. Let $x_{0_{1}}, x_{0_{2}}, \cdots, x_{0_{p(0)}}$ be a basis for $\mathrm{g}^{(0)}$, and for each $j$, positive and negative, let $x_{j_{1}} x_{j_{2}}, \cdots x_{j_{p(j)}}$ be a basis for $g^{\left(r_{j}\right)}$. Give $g$ the metric for which the above basis is orthonormal. We write $|y|$ for the length of an element $y \in g$. Any $y \in \mathfrak{g}$ has a unique decomposition $y=y_{+}+y_{0}+y_{-}$, with $y_{+} \in \mathfrak{g}^{+}, y_{0} \in \mathfrak{g}^{(0)}$ and $y_{-} \in \mathfrak{g}^{-}$. Then $|y|^{2}=\left|y_{+}\right|^{2}+\left|y_{0}\right|^{2}+\left|y_{-}\right|^{2}$.

Let $\boldsymbol{D}$ be the family of all partial derivatives for the given basis. We write $D_{n i}$ for the partial derivative

$$
\frac{\partial^{n_{j_{1}}}}{\partial x_{j_{1}}} \frac{\partial^{n_{j_{2}}}}{\partial x_{j_{2}}} \cdots \frac{\partial^{n_{j_{m}}}}{\partial x_{j_{m}}}
$$

where $n_{j}=\left(n_{j_{1}}, n_{j_{\varepsilon}}, \cdots, n_{j_{m}}\right)$, with $n_{j_{i}} \geqq 0$ for all $i$, for all $j$, positive, negative or zero. Any $D \in \boldsymbol{D}$ can be written $D_{n_{-k}} \cdots D_{n_{k}}$. The order of $D_{n_{j}}, O\left(D_{n_{j}}\right)$, is defined by

$$
O\left(D_{n_{j}}\right)=\sum_{i=1}^{m} n_{j_{i}}
$$

Then the order of $D$ is defined by

$$
O(D)=\sum_{j=-k}^{k} O\left(D_{n_{j}}\right)
$$

The height of $D, h(D)$ is defined by

$$
h(D)=\sum_{j=-k}^{h} r_{j} O\left(D_{n_{j}}\right)
$$

For any real $t$, let $a_{t}=\exp t x$, where exp: $\mathfrak{g} \rightarrow G$ is the exponential map. Then $\left\{a_{t}\right\}_{t \in \boldsymbol{R}}$ is a one-parameter subgroup; we shall need only 
the invariance of $f$ under $\left\{a_{t}\right\}$.

Then if $y \in \mathrm{g}^{\left(r_{j}\right)}, a_{t} \cdot y=e^{t r_{j}} y$, where $a_{t} \cdot y$ denotes the adjoint action of $a_{t}$ on the element $y \in g$. The following lemma shows how the partial derivatives transform under the action of $a_{t}$.

Lemma 2. $D f\left(a_{t} \cdot y\right)=e^{-t h(D)} D f(y)$ for $y \in \mathfrak{g}, y \neq 0$, for any $D \in D$.

Proof. We prove the formula by induction on the order of $D$. Suppose first that $D=\partial / \partial x_{j_{s}}$. Then

$$
\begin{aligned}
\frac{\partial f}{\partial x_{j_{s}}}\left(a_{t} \cdot y\right) & =\lim _{\varepsilon \rightarrow 0} \frac{f\left(a_{t} \cdot y+\varepsilon x_{j_{s}}\right)-f\left(a_{t} \cdot y\right)}{\varepsilon}, \\
& =\lim _{\varepsilon \rightarrow 0} \frac{f\left(y+\varepsilon a_{t}^{-1} \cdot x_{j_{s}}\right)-f(y)}{\varepsilon},
\end{aligned}
$$

by the invariance of $f$,

$$
\begin{aligned}
& =\lim _{\varepsilon \rightarrow 0} \frac{f\left(y+\varepsilon e^{-t r_{j}} x_{j_{s}}\right)-f(y)}{\varepsilon} \\
& =e^{-t r_{j}} \frac{\partial f}{\partial x_{j_{s}}}(y)=e^{-\operatorname{th}(D)} D f(y) .
\end{aligned}
$$

Now assume the lemma is true whenever $O(\bar{D})<k$. If $O(D)=k$, then $D=\left(\partial / \partial x_{j_{l}}\right) \bar{D}$, where $O(\bar{D})=k-1$.

$$
\begin{aligned}
D f\left(a_{t} \cdot y\right) & =\lim _{\varepsilon \rightarrow 0} \frac{\bar{D} f\left(a_{t} \cdot y+\varepsilon x_{j_{l}}\right)-\bar{D} f\left(a_{t} y\right)}{\varepsilon} \\
& =\lim _{\epsilon \rightarrow 0} e^{-t h(\bar{D})} \frac{\left(\bar{D} f\left(y+\varepsilon a_{t}^{-1} \cdot x_{j_{l}}\right)-\bar{D} f(y)\right)}{\varepsilon} \\
& =e^{-t h(\bar{D})} \lim _{\epsilon \rightarrow 0} \frac{\bar{D} f\left(y+\varepsilon e^{-t r} x_{j_{l}}\right)-\bar{D} f(y)}{\varepsilon} \\
& =e^{-t h(\bar{D})} e^{-t r_{j}} \frac{\partial}{\partial x_{j_{l}}} D f(y) \\
& =e^{-t h(D)} D,
\end{aligned}
$$

which proves Lemma 2.

Lemma 3. Let $y \in \mathfrak{g}-\{0\}$ and $\delta>0$ such that $|y|<\delta$. Then for $D \in \boldsymbol{D}$ and any $\varepsilon>0$, there exists $y^{\prime} \in \mathfrak{g},\left|y^{\prime}\right|<\delta$, such that

$$
y_{+}^{\prime} \neq 0 \text { and } y_{-}^{\prime} \neq 0
$$

and

$$
\left|D f(y)-D f\left(y^{\prime}\right)\right|<\varepsilon .
$$

Proof. Since $D f$ is continuous at $y$, there exists a neighborhood 
$V$ of $y$ such that $z \in V$ implies $|D f(y)-D f(z)|<\varepsilon$. The intersection of $V$ with the ball of radius $\delta$ around the origin is again a neighborhood, $V^{\prime}$, of $y$. Since $|y|^{2}=\left|y_{+}\right|^{2}+\left|y_{0}\right|^{2}+\left|y_{-}\right|^{2}$, if either $y_{+}$or $y_{-}$is 0 , we may choose $z_{+}^{\prime} \in \mathfrak{g}^{+}$and $z_{-}^{\prime} \in \mathfrak{g}^{-}$sufficiently small so that $y^{\prime}=y+$ $z_{+}^{\prime}+z_{-}^{\prime}$ is still in $V^{\prime}$ which proves the lemma.

We now define a compact neighborhood $U$ of width $1 / 2$ around the unit sphere, i.e.,

$$
U=\{y \in g|1 / 2 \leqq| y \mid \leqq 3 / 2\} .
$$

Since $U$ is compact, for any $D \in D, \varepsilon>0$, there exists $\delta_{D}>0$ such that

$$
\left|y_{1}-y_{2}\right|<\delta_{D} \Longrightarrow\left|D f\left(y_{1}\right)-D f\left(y_{2}\right)\right|<\varepsilon \text { for any } y_{1}, y_{2} \in U \text {. }
$$

Lemma 4. Let $D \in \boldsymbol{D}$ with $h(D) \neq 0$. Then for any $\varepsilon>0$, there exists a neighborhood $S_{D}$ of 0 in $g$ such that if $y \in S_{D}-\{0\}$, then $|D f(y)|<\varepsilon$.

Proof. Let $M=\max _{z \in U}|D f(z)|$, Then for any $t \in R, z \in U$ $\left|D f\left(a_{t} \cdot z\right)\right| \leqq\left|e^{-t h(D)} M\right|$, by Lemma 2. We shall assume, to minimize notation, that $h(D)>0$. The proof for $h(D)<0$ is similar. Choose $t_{1}$ satisfying $\left|e^{-t_{1} h(D)} M\right|<\varepsilon$. Then $\left|e^{-t h(D)} M\right|<\varepsilon$ for all $t>t_{1}$. Now let $r=\max _{j=1,2 \cdots k} r_{j}$, and let $\delta_{D}=\min \left(1 / 2, e^{-t_{1} l} r\right)$. We define $S_{D}$ as the sphere of radius $\delta_{D}$, and we shall show that this satisfies our condition. For suppose $y=y_{+}+y_{0}+y_{-} \in S_{D}$. By Lemma 3 it suffices to assume that $y_{+} \neq 0$ and $y_{-} \neq 0$. Since $|y|^{2}=\left|y_{+}\right|^{2}+\left|y_{0}\right|^{2}+\left|y_{-}\right|^{2}$, we have $\left|y_{-}\right|<\delta_{D}$. Since $y_{-} \neq 0$, there exists $t$ such that $\left|a_{-t} \cdot y\right|=1$. Then

$$
\left|a_{-t} \cdot y-a_{-t} \cdot y_{-}\right|=\left|a_{-t} \cdot\left(y_{0}+y_{+}\right)\right| \leqq\left|y_{0}+y_{+}\right|<\delta_{D} \leqq \frac{1}{2} .
$$

So that $a_{-t} \cdot y \in U$. But

$$
1=\left|a_{-t} \cdot y_{-}\right| \leqq|r|\left|e^{t}\right|\left|y_{-}\right|,
$$

so that

$$
e^{t} \geqq 1 / r\left|y_{-}\right|>1 / r\left(e^{-t_{1}} / r\right)=e^{t_{1}},
$$

which proves that $t>t_{1}$. Therefore, since $a_{-t} \cdot y \in U$, it follows from the definition of $t_{1}$ that $\left|D f\left(a_{t} \cdot\left(a_{-t} \cdot y\right)\right)\right|<\varepsilon$. Since $a_{t} \cdot\left(a_{-t} \cdot y\right)=y$, this proves Lemma 4.

Lemma 5. For any $D \in \boldsymbol{D}$ with $h(D)=0$ and any $\varepsilon>0$, there exists a neighborhood $S_{D}$ of 0 in $g$ such that if $y, y^{\prime} \in S_{D}-\{0\}$, then $\left|D f(y)-D f\left(y^{\prime}\right)\right|<\varepsilon$. 
Proof of Lemma 5. Choose $\delta, 0<\delta \leqq 1$, such that for any $z, z^{\prime} \in U$,

$$
\left|z-z^{\prime}\right|<\delta \text { implies }\left|D f(z)-D f\left(z^{\prime}\right)\right|<\frac{\varepsilon}{5},
$$

and let $S_{D}$ be the ball of radius $\delta / 2$ around 0 . Now let $y, y^{\prime} \in S_{D}$ be arbitrary. We will show that $\left|D f(y)-D f\left(y^{\prime}\right)\right|<\varepsilon$.

We write

$$
y=y_{+}+y_{0}+y_{-}
$$

and

$$
y^{\prime}=y_{+}^{\prime}+y_{0}^{\prime}+y_{-}^{\prime}
$$

as before. By Lemma 3 we may assume that $y_{+}, y_{-}, y_{+}^{\prime} y_{-}^{\prime}$ are all nonzero. We show first

$$
\left|D f\left(y_{0}+y_{+}\right)-D f(y)\right|<\frac{\varepsilon}{5} \text { and }\left|D f\left(y_{0}^{\prime}+y_{+}^{\prime}\right)-D f\left(y^{\prime}\right)\right|<\frac{\varepsilon}{5} .
$$

For this, choose $t>0$ such that $\left|a_{t} \cdot y_{+}\right|=1$. As in the proof of Lemma 4, $a_{t} \cdot y$ and $a_{t} \cdot\left(y_{0}+y_{+}\right) \in U$. Then $\left|a_{t} \cdot y-a_{t} \cdot\left(y_{0}+y_{t}\right)\right|=$ $\left|a_{t} \cdot y_{-}\right|<\left|y_{-}\right|<\delta$. By the choice of $\delta$, we have $\mid D f\left(a_{t} \cdot y\right)-D f\left(a_{t} \cdot\left(y_{0}+\right.\right.$ $\left.\left.y_{+}\right)\right)<\varepsilon / 5$. Since $h(D)=0, D f$ is invariant under $a_{t}$, so that the first inequality of (3) holds. The proof of the second is the same.

By continuity of $D f$ at $y_{0}+y_{+}$and $y_{0}^{\prime}+y_{+}^{\prime}$, we may choose $q_{-} \epsilon$ $\mathfrak{g}^{-}$with $\left|q_{-}\right|$sufficiently small so that

$$
\left|D f(\bar{y})-D f\left(y_{0}+y_{+}\right)\right|<\varepsilon / 5 \text { and }\left|D f\left(\bar{y}^{\prime}\right)-D f\left(y_{0}^{\prime}+y_{+}^{\prime}\right)\right|<\varepsilon / 5,
$$

where $\bar{y}=q_{-}+y_{0}+y_{+}$and $\bar{y}^{\prime}=q_{-}+y_{0}^{\prime}+y_{+}^{\prime}$. Now choose $s>0$ such that $\left|a_{-s} \cdot q_{-}\right|=1$, which is possible since $q_{-} \neq 0$. Then $a_{-s} \cdot \bar{y} \in$ $U$ and $a_{-s} \cdot \bar{y}^{\prime} \in U$. We shall show

$$
\left|D f(\bar{y})-D f\left(\bar{y}^{\prime}\right)\right|<\frac{\varepsilon}{5} .
$$

Indeed,

$$
\begin{aligned}
\left|a_{-s} \cdot \bar{y}-a_{-s} \cdot \bar{y}^{\prime}\right| & =\left|a_{-s} \cdot\left(y_{0}+y_{+}\right)-a_{-s} \cdot\left(y_{0}^{\prime}+y_{+}^{\prime}\right)\right| \\
& \leqq\left|a_{-s} \cdot\left(y_{0}+y_{+}\right)\right|+\left|a_{-s}\left(y_{0}^{\prime}+y_{+}^{\prime}\right)\right| \\
& \leqq\left|y_{0}+y_{+}\right|+\left|y_{0}^{\prime}+y_{+}^{\prime}\right| \\
& <\frac{\delta}{2}+\frac{\delta}{2}=\delta,
\end{aligned}
$$

which proves $\left|D f\left(a_{-s} \cdot \bar{y}\right)-D f\left(a_{-s} \cdot \bar{y}^{\prime}\right)\right|<\varepsilon / 5$. Then (5) follows immediately since $D f$ is invariant under $a_{-s}$.

To complete the proof of Lemma 5 , note that 


$$
\begin{aligned}
&\left|D f(y)-D f\left(y^{\prime}\right)\right| \leqq \mid D f(y)+D f\left(y_{0}+y_{+}\right)|+| D f\left(y^{\prime}\right)-D f\left(y_{0}^{\prime}+y_{+}^{\prime}\right) \mid \\
&+\left|D f\left(y_{0}+y_{+}\right)-D f(\bar{y})\right|+\mid D f\left(y_{0}^{\prime}+y_{+}^{\prime}\right) \\
&-D f\left(\bar{y}^{\prime}\right)|+| D f(\bar{y})-D\left(\bar{y}^{\prime}\right) \mid \\
&<\frac{\varepsilon}{5}+\frac{\varepsilon}{5}+\frac{\varepsilon}{5}+\frac{\varepsilon}{5}+\frac{\varepsilon}{5} \text { by (3), (4) and }(5)=\varepsilon .
\end{aligned}
$$

We may now complete the proof of the theorem. Lemmas 4 and 5 show that for any $D \in D$, the function $D f$ can be extended continuously over 0 .

We shall assume the following, which can be proved using elementary calculus.

(6) If $h(x)$ is a function on $\boldsymbol{R}$ such that $d h / d x$ exists and is continuous off 0 , then $h$ is differentiable if the function $d h / d x$ can be extended continuously over 0 .

By (6) and induction, it follows that $D f$ exists and is continuous on all of $\mathfrak{g}$ for any $D \in D$.

This finishes the proof of the theorem.

\section{REFERENCES}

1. S. Helgason, Differential geometry and symmetric spaces, Pure and Applied Mathematics, Vol. XII. Academic Press, New York-London, 1962.

2. L. Hormander, Linear partial differential operators, Die Grundlehren der mathematischen Wissenschaften, Academic Press, Inc. Publishers, New York; Springer-Verlag, Berlin-Gottingen-Heidelberg, 1963.

3. J. J. Kohn and L. Nirenberg, An algebra of pseudo-differential operators, Comm. Pure Appl. Math., 18 (1965), 269-305.

4. B. Kostant, Lie group representations on polynomial rings, Amer. J. Math., 85 (1963), 327-404.

5. J.-P. Serre, Algebres de Lie Semi-Simples Complexes, Benjamin, New York, 1966.

6. H. Stetkaer, Invariant pseudo-differential operators, Math. Scand., 28 (1971), 105123.

7. — Invariant pseudo-differential operators, Thesis, M.I.T (1969).

8. A. Melin, A remark about pseudo-differential operators, (to appear).

Received April 19, 1971 and in revised form September 28, 1971.

TUFTS UNIVERSITY

Present Address: Columbia University 


\section{PACIFIC JOURNAL OF MATHEMATICS}

\section{EDITORS}

\author{
H. SAMELSON \\ Stanford University \\ Stanford, California 94305 \\ C. R. HOBBY \\ University of Washington \\ Seattle, Washington 98105
}

\author{
J. DugundJI \\ Department of Mathematics \\ University of Southern California \\ Los Angeles, California 90007
}

\author{
RICHARD ARENS \\ University of California \\ Los Angeles, California 90024
}

\section{ASSOCIATE EDITORS}
E. F. BECKENBACH
B. H. NeumanN
F. WOLF
K. YoSHIDA

\section{SUPPORTING INSTITUTIONS}

\author{
UNIVERSITY OF BRITISH COLUMBIA \\ CALIFORNIA INSTITUTE OF TECHNOLOGY \\ UNIVERSITY OF CALIFORNIA \\ MONTANA STATE UNIVERSITY \\ UNIVERSITY OF NEVADA \\ NEW MEXICO STATE UNIVERSITY \\ OREGON STATE UNIVERSITY \\ UNIVERSITY OF OREGON \\ OSAKA UNIVERSITY
}

\author{
UNIVERSITY OF SOUTHERN CALIFORNIA \\ STANFORD UNIVERSITY \\ UNIVERSITY OF TOKYO \\ UNIVERSITY OF UTAH \\ WASHINGTON STATE UNIVERSITY \\ UNIVERSITY OF WASHINGTON \\ $* * * *$
$*$
AMERICAN MATHEMATICAL SOCIETY
NAVAL WEAPONS CENTER
}

The Supporting Institutions listed above contribute to the cost of publication of this Journal, but they are not owners or publishers and have no responsibility for its content or policies.

Mathematical papers intended for publication in the Pacific Journal of Mathematics should be in typed form or offset-reproduced, (not dittoed), double spaced with large margins. Underline Greek letters in red, German in green, and script in blue. The first paragraph or two must be capable of being used separately as a synopsis of the entire paper. The editorial "we" must not be used in the synopsis, and items of the bibliography should not be cited there unless absolutely necessary, in which case they must be identified by author and Journal, rather than by item number. Manuscripts, in duplicate if possible, may be sent to any one of the four editors. Please classify according to the scheme of Math. Rev. Index to Vol. 39. All other communications to the editors should be addressed to the managing editor, Richard Arens, University of California, Los Angeles, California, 90024.

50 reprints are provided free for each article; additional copies may be obtained at cost in multiples of 50 .

The Pacific Journal of Mathematics is published monthly. Effective with Volume 16 the price per volume (3 numbers) is $\$ 8.00$; single issues, $\$ 3.00$. Special price for current issues to individual faculty members of supporting institutions and to individual members of the American Mathematical Society: $\$ 4.00$ per volume; single issues $\$ 1.50$. Back numbers are available.

Subscriptions, orders for back numbers, and changes of address should be sent to Pacific Journal of Mathematics, 103 Highland Boulevard, Berkeley, California, 94708.

PUBLISHED BY PACIFIC JOURNAL OF MATHEMATICS, A NON-PROFIT CORPORATION

Printed at Kokusai Bunken Insatsusha (International Academic Printing Co., Ltd.), 270, 3-chome Totsuka-cho, Shinjuku-ku, Tokyo 160, Japan. 


\section{Pacific Journal of Mathematics}

\section{Vol. 43, No. 2 \\ April, 1972}

Arne P. Baartz and Gary Glenn Miller, Souslin's conjecture as a problem on the real line....................................... 277

Joseph Barback, On solutions in the regressive isols ............... 283

Barry H. Dayton, Homotopy and algebraic K-theory ................ 297

William Richard Derrick, Weighted convergence in length ............ 307

M. V. Deshpande and N. E. Joshi, Collectively compact and semi-compact sets of linear operators in topological vector spaces ............. 317

Samuel Ebenstein, Some $H^{p}$ spaces which are uncomplemented in $L^{p} \ldots . .327$

David Fremlin, On the completion of locally solid vector lattices ......... 341

Herbert Paul Halpern, Essential central spectrum and range for elements of

a von Neumann algebra............................... 349

G. D. Johnson, Superadditivity intervals and Boas' test ............. 381

Norman Lloyd Johnson, Derivation in infinite planes . . . . . . . . . . . 387

V. M. Klassen, The disappearing closed set property .............. 403

B. Kuttner and B. N. Sahney, On the absolute matrix summability of Fourier series ........................................... 407

George Maxwell, Algebras of normal matrices................... 421

Kelly Denis McKennon, Multipliers of type $(p, p) \ldots \ldots \ldots \ldots \ldots \ldots . . \ldots 29$

James Miller, Sequences of quasi-subordinate functions ............. 437

Leonhard Miller, The Hasse-Witt-matrix of special projective varieties ..... 443

Michael Cannon Mooney, A theorem on bounded analytic functions ...... 457

M. Ann Piech, Differential equations on abstract Wiener space .......... 465

Robert Piziak, Sesquilinear forms in infinite dimensions ............. 475

Muril Lynn Robertson, The equation $y^{\prime}(t)=F(t, y(g(t))) \ldots \ldots \ldots \ldots .483$

Leland Edward Rogers, Continua in which only semi-aposyndetic

subcontinua separate ............................... 493

Linda Preiss Rothschild, Bi-invariant pseudo-local operators on Lie

groups ...................................... 503

Raymond Earl Smithson and L. E. Ward, The fixed point property for

arcwise connected spaces: a correction ...................... 511

Linda Ruth Sons, Zeros of sums of series with Hadamard gaps .......... 515

Arne Stray, Interpolation sets for uniform algebras............... 525

Alessandro Figà-Talamanca and John Frederick Price, Applications of random Fourier series over compact groups to Fourier multipliers .. 